\title{
Why Do Patients With Systemic Lupus Erythematosus Suffer Pain?
}

\author{
Anisur Rahman ${ }^{1}$ (ID
}

Systemic lupus erythematosus (SLE) is a systemic autoimmune disease with a prevalence of approximately 1 in $1000 .{ }^{1}$ It occurs 9 times more commonly in women than men and is more common in some ethnic groups, notably in people of African/Caribbean ethnicity. SLE can affect any organ or tissue in the body and patients therefore suffer a wide range of symptoms. However, pain is one of the most frequent and most troublesome symptoms in patients with this disease. ${ }^{2}$ To some extent, pain in SLE can be ascribed to localized inflammation of particular organs, for example, joint inflammation, pleurisy, or pericarditis. Much of the pain in patients with SLE, however, is more generalized than this. That type of chronic generalized pain is less likely to respond to immunosuppressive treatment targeted to inflammation of specific organs.

In fact, chronic pain is a societal problem. Multiple studies in different countries have shown that the prevalence of chronic pain (often defined as being pain every day for at least 3 months) is as high as $30 \%,{ }^{3,4}$ whereas chronic widespread pain (both sides of the body, above and below the waist, and in the spine) is found in $10-11 \%$ of the population. ${ }^{3,4,5}$ Studies have shown that psychological and social factors play a key role in both onset ${ }^{6}$ and persistence of chronic pain ${ }^{7}$ in the population. This leads to the biopsychosocial model of pain, implying that addressing the biological and physical causes of pain alone will be insufficient to control this symptom and its deleterious effects on quality of life. ${ }^{8}$

In patients with SLE, therefore, it is important to investigate how much of the burden of pain is directly related to disease-associated factors and how much is due to generic psychological and social factors. Further, these 2 types of factors can be linked: a patient suffering from highly active SLE may consequently

${ }^{1}$ A. Rahman, PhD, FRCP, Professor of Rheumatology, Centre for Rheumatology Research, Division of Medicine, University College London, London, UK.

The author declares no conflicts of interest.

Address correspondence to Dr. A. Rahman, Centre for Rheumatology Research, Room 412, Rayne Building, 5 University Street, London WC1E 6JF, UK.

Email:anisur.rahman@ucl.ac.uk. experience anxiety, depression, and difficulties with socioeconomic issues such as employment and personal relationships.

Disease activity and damage are separate facets of SLE, and both could contribute to pain. Disease activity implies current inflammation, which may be reversible with treatment and is typically quantified using validated disease activity scores such as the Systemic Lupus Disease Activity Index (SLEDAI), Systemic Lupus Activity Measure (SLAM), or the British Isles Lupus Assessment Group (BILAG) index. Damage implies irreversible change due to either the disease or treatment and is measured using the Systemic Lupus International Collaborative Clinics Damage Index (SLICC-DI).

Therefore, in trying to quantify the contribution of damage and activity to the experience of pain in patients with SLE, it is necessary to investigate a sample of patients in whom activity, damage, pain, and other potential contributing factors are all measured simultaneously, such that a comprehensive statistical analysis can be carried out. A major problem, however, is that while pain research is typically carried out on large samples of subjects using patient-reported questionnaires, this approach is not ideally suited for measuring disease activity or damage in patients with SLE. SLEDAI, SLAM, BILAG, and SLICC-DI are complex instruments that require face-to-face assessment by a professional as well as laboratory assays. In an attempt to resolve the difficulty of measuring activity by means of patient-reported questionnaires, Karlson, et al developed the Systemic Lupus Activity Questionnaire (SLAQ). ${ }^{9}$ SLAQ is based on SLAM but includes only those elements that can be self-assessed by patients. It has been validated and shown to have reasonable correlation with SLAM assessed at the same time $(r=0.62) .{ }^{9}$ Similarly, the Brief Index of Lupus Damage (BILD) is a patient-reported questionnaire that includes a subset of the elements of SLICC-DI and has been validated. ${ }^{10} R$ value for correlation between BILD and SLICC-DI was $0.64 .^{10}$

In this issue of The Journal of Rheumatology, Falasinnu, et al report on a cross-sectional analysis of patient-reported data from 766 patients with SLE. ${ }^{11}$ These patients are members of

\section{See Pain in SLE, page 1279}


the Georgians Organized Against Lupus (GOAL) cohort and complete annual research surveys. In these surveys, disease activity and damage were assessed by SLAQ and BILD, respectively, whereas average pain intensity over the previous 7 days was measured on an ordinal scale from 0 to 10 , and pain interference with daily activities was measured using a 5-point Likert scale. Importantly, use of the patient-reported survey method enabled collection of a wealth of other relevant data regarding socioeconomic, psychological, and behavioral factors. Therefore, the authors were able to carry out detailed hierarchical regression analyses and sensitivity analysis (in which pain-related items were removed from the SLAQ score) to delineate the contribution to pain intensity and interference made by SLE activity and damage in comparison to other factors. They found that activity and damage together explained $32 \%$ of the variance in pain intensity and $33 \%$ of the variance in pain interference. In the sensitivity analysis, activity, and damage explained $25 \%$ of the variance in pain intensity and interference. Socioeconomic factors, such as age, male sex, lower income, lower educational attainment, and Black race explained $4-9 \%$ of variance in pain outcomes, whereas psychosocial and behavioral factors accounted for $4 \%$ of this variance. Increasing sleep disturbance and increasing BMI were associated with increased pain intensity and interference.

Previous authors have reported on the importance of pain as a symptom in patients with SLE. Pettersson, et al explored the opinions of 324 Swedish patients as to the symptoms that they found most distressing and reported that $50 \%$ identified pain as their most difficult symptom ever, whereas $32 \%$ said that it was the most difficult current symptom. ${ }^{2}$ Waldheim and colleagues subdivided a group of 84 patients with SLE into 20 with high self-reported pain intensity and 64 with low intensity. ${ }^{12}$ The high pain group had higher fatigue, depression, and anxiety, and lower quality of life. They had only mild/moderate disease activity (though significantly higher activity than the low pain group). No previous group, however, has carried out a detailed analysis of the biological, social, and psychological factors contributing to pain in SLE prior to the paper of Falasinnu, et $a l^{11}$ in this issue.

Describing limitations of their work, the authors note that their study is cross-sectional and is therefore unable to comment on causality; also, they did not collect data on fibromyalgia (FM). It seems likely that FM could have been the cause of pain in some of these subjects since it has an estimated prevalence in the United States of $6.4 \%,{ }^{13}$ and $36.7 \%$ of 439 patients with SLE fulfilled classification criteria for FM in a study by Wolfe, et al. ${ }^{14}$ Since FM can be diagnosed by a questionnaire using the American College of Rheumatology 2010 criteria, ${ }^{15}$ it would have been possible to obtain this information using patient-reported surveys; however, this would not have altered the main messages of the paper.

What messages can patients with SLE and the healthcare professionals treating them take from this paper? First, a significant proportion of pain in these patients is related to disease activity and damage. Since it is also known that persistent activity predisposes to subsequent damage, ${ }^{16}$ this implies that optimising treatment to minimize disease activity can play some role in reducing pain in patients with SLE. It is equally important, however, to recognize that a larger proportion of pain in patients with SLE is not directly explicable by disease-associated factors. Rather, pain in these patients is multifactorial and must be addressed by a variety of psychological, social, and behavioral interventions. Based on their results, the authors comment particularly on obesity and sleep hygiene. They also note a potential role for nonpharmacological self-management programs. Such programs have the advantage of being safe and inexpensive and can be delivered to large numbers of people in the community, though it is often difficult to show statistically significant effects on pain intensity or interference. ${ }^{17}$

\section{REFERENCES}

1. Rees F, Doherty M, Grainge M, Davenport G, Lanyon P, Zhang W. The incidence and prevalence of systemic lupus erythematosus in the UK, 1999-2012. Ann Rheum Dis 2016;75:136-41.

2. Pettersson S, Lovgren M, Eriksson LE, Moberg C, Svenungsson E, Gunnarsson I, et al. An exploration of patient-reported symptoms in systemic lupus erythematosus and the relationship to health-related quality of life. Scand J Rheumatol 2012;41:383-90.

3. Wolfe F, Ross K, Anderson J, Russell IJ, Hebert L. The prevalence and characteristics of fibromyalgia in the general population. Arthritis Rheum 1995;38:19-28.

4. Bergman S, Herrstrom P, Hogstrom K, Petersson IF, Svensson B, Jacobsson LT. Chronic musculoskeletal pain, prevalence rates, and sociodemographic associations in a Swedish population study. J Rheumatol 2001;28:1369-77.

5. Papageorgiou AC, Silman AJ, Macfarlane GJ. Chronic widespread pain in the population: a seven year follow up study. Ann Rheum Dis 2002;61:1071-4.

6. McBeth J, Macfarlane GJ, Benjamin S, Silman AJ. Features of somatization predict the onset of chronic widespread pain: results of a large population-based study. Arthritis Rheum 2001;44:940-6.

7. McBeth J, Macfarlane GJ, Hunt IM, Silman AJ. Risk factors for persistent chronic widespread pain: a community-based study. Rheumatology 2001;40:95-101.

8. Foster NE, Pincus T, Underwood MR, Vogel S, Breen A, Harding G. Understanding the process of care for musculoskeletal conditions--why a biomedical approach is inadequate. Rheumatology 2003;42:401-4.

9. Karlson EW, Daltroy LH, Rivest C, Ramsey-Goldman R, Wright EA, Partridge AJ, et al. Validation of a Systemic Lupus Activity Questionnaire (SLAQ) for population studies. Lupus 2003; 12:280-6.

10. Yazdany J, Trupin L, Gansky SA, Dall'era M, Yelin EH, Criswell LA, et al. Brief index of lupus damage: a patient-reported measure of damage in systemic lupus erythematosus. Arthritis Care Res 2011;63:1170-7.

11. Falasinnu T, Drenkard C, Bao G, Mackey S, Lim SS. The problem of pain in lupus: an explication of the role of biopsychosocial mechanisms. J Rheumatol 2021;48:1279-89.

12. Waldheim E, Elkan AC, Pettersson S, van Vollenhoven R, Bergman S, Frostegard J, et al. Health-related quality of life, fatigue and mood in patients with SLE and high levels of pain compared to controls and patients with low levels of pain. Lupus 2013;22:1118-27.

13. Vincent A, Lahr BD, Wolfe F, Clauw DJ, Whipple MO, Oh TH, et al. Prevalence of fibromyalgia: a population-based study in Olmsted County, Minnesota, utilizing the Rochester Epidemiology Project. Arthritis Care Res 2013;65:786-92. 
14. Wolfe F, Clauw DJ, Fitzcharles MA, Goldenberg DL, Hauser W, Katz RS, et al. Fibromyalgia criteria and severity scales for clinical and epidemiological studies: a modification of the ACR Preliminary Diagnostic Criteria for Fibromyalgia. J Rheumatol 2011;38:1113-22.

15. Wolfe F, Clauw DJ, Fitzcharles MA, Goldenberg DL, Katz RS, Mease P, et al. The American College of Rheumatology preliminary diagnostic criteria for fibromyalgia and measurement of symptom severity. Arthritis Care Res 2010;62:600-10.
16. Sutton EJ, Davidson JE, Bruce IN. The systemic lupus international collaborating clinics (SLICC) damage index: a systematic literature review. Semin Arthritis Rheum 2013;43:352-61.

17. Taylor SJ, Carnes D, Homer K, Kahan BC, Hounsome N, Eldridge $S$, et al. Novel three-day, community-based, nonpharmacological group intervention for Chronic Musculoskeletal Pain (COPERS): a randomised clinical trial. PLoS Med 2016;13:e1002040. 